\title{
PARTICIPAÇÃO DOS ALIMENTOS NO CONSUMO DIÁRIO COMO FONTE DE NUTRIENTES E DO MICRONUTRIENTE CÁLCIO, NO GRUPO DE IDOSOS ESTUDADOS DO MUNICÍPIO DE CURITIBA (PARANÁ/BR).
}

\section{THE PARTICIPATION OF FOOD ON DAILY COMSUPTION AS A SOURCE OF CALCIUM AND ENERGY NUTRIENTS OF ELDERLY GROUP FROM CURITIBA CITY (PARANÁ/BR)}

\author{
PENTEADO', P. T. P. da S.; ALMEIDA², D. M. de; SOARES², J. J.; OLIVEIRA ${ }^{3}$, K. S.
}

\begin{abstract}
1 Docente Doutor na Disciplina de Tecnologia de Alimentos Dietéticos,UFPR, ppenteado@ufpr.br 2 Farmacêutica Industrial pela UFPR, bolsistas de iniciação científica durante a graduação.
\end{abstract} 3 Docente Especialista na Faculdade Espírita (Paraná)

Recebido em: 07/2004 Aprovado em: 08/2004

\begin{abstract}
RESUMO
O consumo alimentar tem sido objeto de estudo sob vários aspectos e tem apresentado importante modificação, sendo explicada ora pelo próprio modelo agro-industrial brasileiro instituído ora pelo comportamento da vida urbana ora pelo nível de transferência das informações à população sobre os cuidados com a saúde e qualidade de vida. O presente estudo é o resultado de uma extensa pesquisa sobre os hábitos e consumo alimentar que, entre os dados, evidencia a participação de diversos alimentos na dieta de idosos ( $e^{\prime \prime} 60$ anos)selecionados em três bairros com diferentes condições de vida de Curitiba. O objetivo buscou identificar a participação e a quantificação dos alimentos no consumo diário como fonte dos nutrientes cálcio e energia. Entre os alimentos com importante participação na alimentação diária e fornecedores de cálcio foram identificados o espinafre, iogurte, pão francês, couve manteiga, mamão e leite e seus derivados. Enquanto que os alimentos consumidos que garantem a energia diária aos idosos foram o arroz branco, biscoitos, bisteca de porco, chocolate, frango assado, margarina, pão forma, vinho tinto além do açúcar refinado, leite e derivados e o pão francês. O perfil de consumo permite identificar estratégias a adequação nutricional considerando os hábitos alimentares dos idosos, como também indica o potencial ao desenvolvimento tecnológico de produtos alimentícios e alternativos nutricionais mais adequados e aceitos pelo consumidor. E, assim melhorar a qualidade nutricional da oferta de alimentos aos idosos.
\end{abstract}

PALAVRAS-CHAVE: consumo alimentar, alimentos, idoso, qualidade de vida.

\begin{abstract}
The food consumption has been an object of study by a lot of aspects and presented as an important modification, explained sometimes by the own Brazilian agro business, sometimes by the behavior of the urban life, and sometimes explained by the of information of the population, about health care and life quality. This study is the result of a wide research about the habits and the food consumption that points the participation of a lot of nutrients on the diet of aged people (e" 60 years) from three neighborhoods with different kind of life conditions from Curitiba, Brazil. The principal intention was to identify the participation and quantification of the food from the daily consumption as a source of the calcium and energy nutrients. Among all foods with an important participation on the daily consumption and the calcium sources were spinach, yogurt, bread, cabbage, papaya and milk and dairy products. The food that guarantees the daily allowance of energy to the elderly people were the white rice, cookies, pork meat, chocolate, roast chicken, bread, butter, wine, sugar, milk and dairy products. The consumption profile allows to identifier some strategies to the nutritional adequation considering the habits of the elderly, as indicates the potential to the technology development of the food products and nutritional alternatives more adequate and accepted by the consumer and with this, improve the nutritional quality of the food offer to the elderly.

Key words: food consumption, food, quality of life.
\end{abstract}

\section{INTRODUÇÃO}

O consumo alimentar tem sido analisado sob vários aspectos, apresentando uma dinâmica de modificações e diferenciações, explicadas pelo próprio modelo agro-industrial brasileiro instituído ou pelo comportamento da vida urbana ou pelo nível de transferência das informações à população sobre os cuidados com a saúde e qualidade de vida. Tais fatores têm determinado modos de consumo diferenciados e, diretamente, afetado por 
fatores de ordem econômica, social, cultural, nutricional e outros, cujos valores obtidos tem conduzido à coexistência de altas prevalências de desnutrição energético - protéica e de obesidade. De modo que os riscos alimentares e seus agravos, na saúde do brasileiro, tem sido relacionados à ingestão de uma dieta qualitativamente inadequada quanto da falta absoluta de acesso de alimentos (MALUF et al., 1996).

Neste contexto alimentar, em paralelo, estão ocorrendo mudanças no perfil demográfico as quais indicam novas perspectivas de padrão alimentar e cuidados aos grupos com necessidades dietéticas específicas (crianças, adolescentes, esportistas, idosos, etc). No caso dos idosos, as modificações no consumo de alimentos decorrem também das alterações, que acontecem no envelhecimento, como as metabólicas, físicas (como a mastigação) ou fisiológicas (como por medicação, perda da sensação de sede) à alimentação e das restrições no preparo das refeições, na compra de alimentos assim como no modo de compartilhar as refeições e os alimentos no ambiente familiar (COITINHO et al., 1991).

VELLAS (1992) e DUARTE (2002) associam o envelhecimento com um declínio fisiológico e aumento de doenças, acidentes e situações de estresse (tal como ocorre quando se associa o declínio da densidade óssea e o aumento da percentagem de gordura com a morbidade relacionada à osteoporose e doenças cardiovasculares (ROSENBERG e MILLER, 1992).

Sob o aspecto nutricional, tanto a obesidade como a desnutrição tem extensas conseqüências para o idoso. A primeira porque potencializa algumas doenças que os idosos, mesmo não obesos, apresentam com maior freqüência (como diabetes mellitus, hipertensão arterial, dislipidemias, doenças cardiovasculares e câncer). A desnutrição protéica - calórica, por sua vez, é o distúrbio nutricional mais importante observado nos idosos, estando associada ao aumento da mortalidade e da susceptibilidade às infecções e à redução da qualidade de vida (OTERO, 2002). Os maiores riscos de desnutrição no idoso são: a) a ingestão de menos de oito refeições principais por semana; b) o consumo muito baixo de leite; c) o consumo negligenciado de frutas e verduras; c) longos períodos durante o dia sem se alimentar ou ingerir líquidos; d) depressão e solidão; e) pobreza; f) restrições no preparo das refeições, na compra de alimentos, entre outros (COITINHO et al.,1991; STAEHELIN; 2002).

GALEAZZI et al.(1997) mostraram para a população idosa de Curitiba percentuais com consumo inadequado de nutrientes, tais como: proteína - 14, cálcio - 70, ferro -30, vitamina A - 3, 1, vitamina C - 8,0, gordura - 49, ácidos graxos saturados - 51, colesterol - 34. PENTEADO et al. (2001) também indicaram inadequação alimentar num grupo selecionado de idosos do mesmo Município e, quando relacionada à renda familiar, apontaram diferenças principalmente no tipo de alimento consumido tal como, por exemplo, para os idosos com renda menor havia o menor consumo de fontes protéicas - frango e carne bovina. Contudo, mesmo os idosos incluídos nas faixas de renda maiores apresentaram inadequação nutricional quando aumentaram o consumo de fontes de carboidratos em substituição dos lipídios (PENTEADO, 2001).

Estes estudos servem a constatar a afirmação de que a pobreza é a origem da maior parte das carências nutricionais, face às condições de consumo de alimentos, essencialmente, calóricos e desprovidos de proteínas de alto valor biológico, de sais minerais e vitaminas (ALNWICK, 1993, p.5). E, quando este quadro resultante de consumo passa a ser relacionado à condição do envelhecimento biológico, as repercussões nutricionais são maiores. 
Em relação ao nutriente cálcio, a alta inadequação de seu consumo foi identificada por GALEAZZl et al. (1997) e por PENTEADO (2001) para idosos curitibanos, demonstrando baixo consumo de alimentos fonte deste micronutriente e necessidade de medidas preventivas aos riscos associados ao consumo. As modificações que ocorrem no envelhecimento fazem com que os idosos constituam um grupo de risco quando se trata de deficiência de cálcio, pois associando a absorção reduzida de cálcio, a diminuição dos níveis de esteróides sexuais com alterações de absorção e de síntese de vitamina $D$ e de seu metabólito ativo, tem-se a formação de um quadro de propensão a hipocalcemia (em especial nas mulheres), a várias condições crônicas, incluindo câncer de cólon, de mama, hipertensão e demência. Um importante fator, que afeta a homeostase do cálcio, está relacionado ao consumo de medicamentos, não apenas qualitativo, mas a concentração dos fármacos e a polifarmácia (MORLEY et al., 1995; PENTEADO, 2001).

Em PENTEADO (2001) foi mencionado que os idosos consomem os mesmos alimentos apenas com variações de freqüência e na forma de apresentação da mesma matéria prima. Entre os alimentos foram mencionados os básicos de consumo da população em geral e também componentes da cesta básica de alimentos (BRASIL, 1938); sendo o arroz (e massas), feijão, carne, acompanhados por hortaliças e frutas. Estes mesmos alimentos foram identificados com algumas variações na ordenação e na contribuição em termos calóricos e protéicos em estudos de SICHIERI et al. (199?) como o arroz, farinha de trigo e biscoitos, açúcar, feijão, banana, laranja, carnes, ovos, leite, óleos, tomate, batata, farinhas de milho e mandioca.

De acordo com a lista de alimentos padronizada em GALEAZZI et al. (1997), UNIVERSIDADE... (1996?), SICHIERI (1998), os grupos de alimentos mais mencionados de consumo entre os idosos, agrupados em bairros, foram os produtos alimentícios à base de cereais entre $21-26 \%$ dos idosos, as verduras em pelo menos $27-29 \%$, as frutas em $13-15 \%$; as carnes e derivados e ovos em 11-15\%; os lácteos em 5-7\% dos entrevistados. Os outros grupos de alimentos - gorduras, doces, bebidas alcoólicas ou não - apresentaram menores participações na alimentação do idoso (PENTEADO, 2001). O consumo diário destes alimentos correspondeu, em energia total, a 1818Kcal para o Bairro Alto, $1563 \mathrm{Kcal}$ para o bairro do Cajuru e 1720 Kcal para o Centro, sendo considerado adequado apesar das necessidades calóricas individuais serem distintas segundo o gênero, idade, atividades físicas, condições de saúde e de nutrição (PENTEADO, 2001).

Em relação aos macronutrientes, os idosos do Cajuru consumiam menores conteúdos de proteínas e de carboidratos, sendo compensado o consumo calórico por lipídios na alimentação diária. O grupo do bairro do Centro tem uma maior contribuição de carboidratos e menor de lipídios na alimentação diária, quando comparada aos outros bairros. Estes resultados indicam ainda que em média os idosos do Bairro Alto tem nas suas refeições diárias o consumo maior de alimentos constituídos de carboidratos e lipídios (PENTEADO, 2001). Em relação ao micronutriente Cálcio, o estudo identificou que o consumo qualitativo de alimentos diários indicou diferenças entre os idosos quando agrupados pelo bairro residencial. O grupo do Centro apresentou valores de 493,01 mg $\pm 313,08$, o do Bairro Alto de $770,30 \mathrm{mg} \pm 528,44$ e o do Cajuru 459,13 mg $\pm 351,46$. Os resultados apresentados por PENTEADO (2001) indicaram inadequação de consumo para os macronutrientes, seja acima do recomendado como para o lipídeo ou abaixo para a proteína nos bairros Cajuru e Bairro Alto. Para o cálcio, o consumo apresentou estar abaixo do recomendado em todos os grupos e, principalmente, para os grupos do Centro e Cajuru. 
Sob o aspecto do risco nutricional, a análise dos dados do estudo de PENTEADO (2001) possibilita apontar a qualidade da alimentação dos idosos. Contudo para qualquer medida nutricional corretiva e eficaz será importante conhecer além a disponibilidade de energia e nutricionais dos alimentos consumidos, a composição alimentar do consumo diário dos idosos - padrão alimentar -, assim como considerar o acesso, os gostos, hábitos, tradições culinárias, representações, práticas, preferências, rituais e tabus. E, assim, sugerir possibilidades para uma alimentação saudável. De modo que para o presente estudo o objetivo foi identificar a participação qualitativa e quantitativa dos alimentos consumidos diariamente como fontes de energia e de cálcio aos idosos selecionados do Município de Curitiba (Paraná).

\section{METODOLOGIA}

A identificação do consumo alimentar em energia e do micronutriente cálcio foi estabelecida a partir dos dados sobre os alimentos preparados e consumidos, correspondendo a 185 alimentos. Eles foram obtidos da entrevista domiciliar com indivíduos com ${ }^{3} 60$ anos de idade, selecionados em 3 bairros do Município de Curitiba com distintas condições de vida (IPPUC, 1996); utilizando o registro em questionário do tipo estruturado e padronizado e, posteriormente, transcrito para meio eletrônico utilizando os softwares EPINFO - 6, WINDOWS 97, EXCELL e VIRTUALNUTRI. Os alimentos foram agrupados por categoria alimentar, identificados quantitativa e qualitativamente em relação aos macronutrientes, energia total e o micronutriente cálcio (PENTEADO, 2001; PENTEADO e OLIVEIRA, 2003) e evidenciada a participação de cada alimento na dieta e a participação de cada alimento como fonte de energia e de cálcio no consumo médio diário. Os alimentos foram agrupados por categoria alimentar e selecionados os alimentos fonte de cálcio ou aqueles constituídos de quantidades de cálcio relevantes o suficiente à comparação.

\section{RESULTADOS}

Segundo a Portaria n 33, da Agência Nacional de Vigilância Sanitária (ANVISA), publicada no dia 13 de janeiro de 1998, Ingestão Diária Recomendada (IDR) é o valor da quantidade de vitaminas, minerais e proteínas que deve ser consumido por dia com o objetivo de atender às necessidades nutricionais da maior parte dos indivíduos e grupos de pessoas de uma população sadia. Considerando o grupo de idosos, objeto de estudo, a IDR de cálcio é de 800mg. Contudo, também é importante observar as orientações da Portaria n 31 da ANVISA, de 13 de janeiro de 1998, sobre alimento "fonte" de um determinado mineral, sendo considerado quando a quantidade de $100 \mathrm{~g}$ possuir pelo menos 15\% da IDR de referência, no caso de sólidos, e 7,5\% da IDR de referência, no caso de líquidos.

A tabela 01 relaciona os alimentos que fizeram parte do estudo e que se enquadram na categoria fonte de cálcio, segundo a legislação, e também alimentos que possuem uma quantidade relevante, isto é que possuam valor próximo a IDR de referência para o cálcio. 
TABELA 01 - Composição centesimal de alimentos fonte de cálcio inseridos na lista do inquérito alimentar.

\begin{tabular}{|c|c|c|c|c|c|c|c|}
\hline ALIMENTOS & ENERGIA (Kcal) & PT (g) & $\mathrm{CH}(\mathrm{g})$ & LIPT (g) & FIBRAS (g) & CÁLCIO (mg) & FERRO (mg) \\
\hline \multicolumn{8}{|l|}{ Cereais e derivados } \\
\hline Pão doce & 237,5 & 7,9 & 45,9 & 2,0 & - & 162,0 & 2,8 \\
\hline Pão francês & 285,6 & 9,4 & 56,8 & 1,6 & 2,8 & 111,0 & 3,1 \\
\hline \multicolumn{8}{|l|}{ Verduras e legumes } \\
\hline Agrião & 11,7 & 2,3 & 1,3 & 0,1 & 3,3 & 118,0 & 0,2 \\
\hline Brócolos & 29,4 & 3,0 & 5,6 & 0,3 & 4,1 & 114,0 & 1,1 \\
\hline Chicória (almeirão) & 23,3 & 1,7 & 4,7 & 0,3 & 0,0 & 100,0 & 0,9 \\
\hline Couve verde & 49,2 & 3,3 & 10,0 & 0,7 & 5,8 & 134,0 & 1,7 \\
\hline Espinafre & 21,4 & 2,9 & 3,5 & 0,4 & 4,1 & 98,9 & 2,7 \\
\hline \multicolumn{8}{|l|}{ Leite e derivados } \\
\hline logurte & 61,2 & 3,5 & 4,7 & 2,1 & 0,0 & 120,0 & 0,1 \\
\hline Leite integral ou A & 64,2 & 3,3 & 4,7 & 3,7 & 0,0 & 119,0 & 0,1 \\
\hline Leite em pó integral & 496,0 & 26,3 & 38,4 & 26,7 & 0,0 & 912,0 & 0,5 \\
\hline Leite do tipo C & 60,6 & 3,3 & 4,6 & 3,0 & 0,0 & 119,0 & 0,1 \\
\hline Queijo & 392,0 & 28,3 & 0,6 & 30,6 & 0,0 & 840,0 & 0,6 \\
\hline Requeijão & 288,0 & 12,4 & 1,3 & 25,0 & 0,0 & 151,0 & 13,3 \\
\hline \multicolumn{8}{|l|}{ Carnes } \\
\hline Camarão & 109,0 & 23,8 & 0,0 & 1,5 & 0,0 & 320,0 & 2,2 \\
\hline Peixe enlatado (sardinha) & 124,0 & 26,0 & 0,0 & 23,2 & 0,0 & 402,0 & 3,2 \\
\hline \multicolumn{8}{|l|}{ Açúcares } \\
\hline Pudim & 294,0 & 9,9 & 41,6 & 10,0 & 0,0 & 243,0 & 0,8 \\
\hline \multicolumn{8}{|l|}{ Bebidas não alcoólicas } \\
\hline Café (solúvel) & 100,0 & 0,5 & 39,5 & 0,6 & 1,0 & 150,0 & 4,0 \\
\hline
\end{tabular}

FONTE: PENTEADO, 2001.

Entre os vários alimentos pode ser evidenciada a importante participação do cálcio na composição do leite e derivados, das hortaliças como o espinafre e a couve manteiga (verde) e do pão francês. No entanto, a TABELA 02 mostra o percentual médio de cálcio fornecido por cada alimento em relação à quantidade total de cálcio ingerida diariamente, conforme os idosos agrupados entre os três bairros e entre os idosos que os consomem. É importante ressaltar que essas médias foram calculadas com base no total de cálcio que cada pessoa consome, não tendo relação direta com níveis de adequação alimentar. 
TABELA 02 - Alimentos agrupados segundo a percentagem média (\%) de consumo do mineral cálcio em relação ao total de cálcio

\begin{tabular}{|c|c|c|}
\hline \multicolumn{3}{|c|}{ Alimentos agrupados segundo a percentagem média (\%) de consumo do mineral Cálcic } \\
\hline Abobrinha frita & Carne bovina gorda & Macarrão \\
\hline Açúcar refinado & Carne bovina magra & Manga \\
\hline Alho & Cebolinha & Melão \\
\hline Batata palha & Cenoura & Pão de centeio \\
\hline Beterraba & Costelinha de porco assada & Pão de queijo \\
\hline Bife & Doces de frutas & Pastel \\
\hline Bisteca de porco assada & Grão de bico & Suco de limão \\
\hline Bom bom sonho de valsa & Lentilha & Tomate \\
\hline Brócolis & & Vagem \\
\hline \multicolumn{3}{|c|}{$1-5,0$} \\
\hline Abóbora & Bolo & Goiaba \\
\hline Abobrinha & Cebola & Mandioca \\
\hline Açúcar mascavo & Cenoura crua & Melancia \\
\hline Agrião & Cerveja & Pão caseiro \\
\hline Alface & Couve flor & Pêra \\
\hline Almeirão & Escarola & Polenta \\
\hline Banana & Farinha & Vinho tinto \\
\hline Berinjela crua & Feijão & \\
\hline \multicolumn{3}{|c|}{$5,1-10$} \\
\hline Almeirão & Repolho & Mandioca \\
\hline Arroz branco & Café preparado & Pão de Forma \\
\hline \multicolumn{3}{|l|}{ Brócolis } \\
\hline & $10,1-20$ & Pão francês \\
\hline Espinafre & logurte & \\
\hline Couve manteiga & Mamão & \\
\hline \multicolumn{3}{|c|}{$>20,1$} \\
\hline Leites diversos & Queijo & \\
\hline
\end{tabular}

Na TABELA 03 estão apresentados os valores relativos ao percentual médio que cada alimento contribui em calorias na dieta de um indivíduo, obtidos na mesma forma que os dados da TABELA 02. Conforme pode ser observado nas Tabelas 2 e 3, há diferentes comportamentos no consumo de energia e de cálcio diário dos alimentos. E se forem analisados dentro de cada grupo as contribuições dos diversos produtos também são evidenciados participações diferentes como no caso do leite e seus derivados ou subprodutos. Quando identificado sob o aspecto de fornecimento de cálcio diário, o leite é responsável por mais de $20 \%$ em média, do total ingerido diariamente, mas participa apenas entre 10 a $20 \%$ do total de calorias ingeridas. Entretanto, alguns alimentos que não são convencionalmente fonte de cálcio possuem contribuição de 10 a 20\% do total de cálcio consumido por dia, como o espinafre, couve manteiga, pão francês e mamão. Estes mesmos alimentos não podem ser considerados fontes de energia por contribuem com menos de $1 \%$ do total de calorias ingeridas diariamente, refletindo um baixo consumo. 
TABELA 03 - Alimentos agrupados segundo a percentagem média (\%) de consumo de calorias em relação ao total de energia

\begin{tabular}{|c|c|c|}
\hline \multicolumn{3}{|c|}{ Alimentos agrupados segundo a percentagem média (\%) de consumo de Calorias } \\
\hline Balas & $\begin{array}{c}<1,0 \\
\text { Fígado de boi }\end{array}$ & Pastel \\
\hline Bombom & Melancia & PeixeTomate \\
\hline \multicolumn{3}{|l|}{ Couve Manteiga } \\
\hline & $1-5,0$ & \\
\hline Abacate & Costela de porco & Mamão \\
\hline Abacaxi & Doce de frutas & Mandioca \\
\hline Açúcar mascavo & Farinha de mandioca crua & Manga \\
\hline Bacon & Feijão & Margarina \\
\hline Bala & Fígado cozido & Pão de centeio \\
\hline Banana & Filé de frango grelhado & Pêra \\
\hline Beterraba & Goiaba & Pipoca doce \\
\hline Bife & logurte & Polenta \\
\hline Biscoito de polvilho & Laranja & Queijos \\
\hline Bolacha agua e sal & Leite condensado & Refrigerante \\
\hline Bolo simples & Leite em pó & Requeijão \\
\hline Brócolis & Leite fermentado & Ricota \\
\hline Café capuccino & Lingüiça de porco & Salsicha \\
\hline Carne $2^{a}$ & Maçã & Sucos de frutas \\
\hline Carne moída refogada & Macarrão & \\
\hline Cerveja & $\begin{array}{l}\text { Maionese } \\
5,1-10\end{array}$ & \\
\hline Arroz branco & Chocolate & Margarina \\
\hline Biscoito & Conhaque & Pão Forma \\
\hline Maisena & Frango assado & Vinho tinto \\
\hline \multicolumn{3}{|l|}{ Biscoito recheado } \\
\hline \multicolumn{3}{|l|}{ Bisteca de porco } \\
\hline & $10,1-20$ & \\
\hline $\begin{array}{l}\text { Açúcar refinado } \\
\text { Leites diversos }\end{array}$ & Pão francês & Pão caseiro \\
\hline
\end{tabular}

No caso do pão francês, ficou enquadrado na categoria de alimentos que foram identificados como sendo responsáveis pelo fornecimento médio diário de 10\% cálcio e de energia. $\mathrm{O}$ arroz branco apresentou importante contribuição como fonte calórica e de cálcio face o nível de consumo, contribui entre 5 a 10\% do total de calorias e de cálcio ingerido em média. Um outro dado relevante é à participação do açúcar refinado como fonte apenas de carboidrato e pobre valor nutricional, porem pouco contribui para uma dieta saudável e em especial aos idosos.

O resultado dessa pesquisa apontando alimentos como espinafre, iogurte, pão francês, couve manteiga, mamão e leite e seus derivados, demonstram a possibilidade de alcançar a adequação das dietas, condicionada aos hábitos alimentares praticados pelos idosos. Ainda que os resultados possam mostrar apenas um dos inúmeros aspectos da nutrição deste grupo de idosos, eles retratam que o consumo de alimentos é uma abordagem norteadora ao diagnóstico e pode servir a inúmeros propósitos (orientação à adequação alimentar desse grupo através da educação, dar subsídios aos programas alimentares, a requalificação teórico - prática do próprio ensino acadêmico, etc.) a fim de garantir qualidade nutricional, melhor estado de saúde e qualidade de vida da população idosa. 


\section{CONCLUSÃO}

- Os alimentos com importante participação na alimentação diária e fornecedores de cálcio, considerando a IDR de 800mg e as especificações legais de fonte de cálcio - o espinafre, iogurte, pão francês, couve manteiga, mamão e leite e derivados.

Os alimentos que garantem a energia diária - açúcar refinado, arroz branco, biscoitos, bisteca de porco, chocolate, frango assado, margarina, pão de forma e vinho tinto, além do açúcar refinado, leite e derivados e o pão francês.

- Importantes fontes de cálcio e energia diária - pão francês e do arroz branco, em face de participação quantitativa no consumo diário.

CONSIDERAÇÕES: O perfil de consumo identificado demonstra a possibilidade de alcançar a adequação alimentar, considerando as estratégias e os hábitos alimentares dos idosos, e permite indicar o potencial ao desenvolvimento tecnológico de produtos alimentícios e alternativos nutricionais mais adequados e aceitos pelo consumidor idoso.

\section{REFERÊNCIAS}

ALNWICK, D. Ferro e ácido fólico: milhões de anemias crônicas. A Prescripção. Washington, n. 8,p.5-9, dez. 1993. ANVISA. AGÊNCIA NACIONAL DE VIGILÂNCIA SANITÁRIA. Portaria n 33 de 13 de janeiro de 1998. Dispões sobre os níveis de Ingestão Diária Recomendada para vitaminas, minerais e proteínas. DOU, Brasil, Brasília, 13 jan. 1999. BRASIL. Decreto-Lei n. 399, de 30 de abril de 1938. Institui as Comissões de Salário Mínimo. DOU, Brasil , Brasília, 7 de maio de 1938, p.8600-8605.

COITINHO, D.C.; LEÃO, M.M.; RECINE, E. e SICHIERI, R. Condições nutricionais da população Brasileira :Adultos e Idosos. Pesquisa Nacional sobre Saúde e Nutrição. Brasília: MS/INAN. 1991

DUARTE, Y. A. de O. O processo de envelhecimento e a assistência ao idoso. Manual de Enfermagem. Disponível em: <http://www.ids-saude.org.br/

enfermagem > Acesso em: 15 jul. 2002.

GALEAZZI, M. A. M et al. Estudo multicêntrico sobre consumo alimentar. Cadernos de Debates, v. especial, 63p. 1997.

IPPUC. INSTITUTO DE PESQUISA E PLANEJAMENTO URBANO DE CURITIBA, Qualidade de Vida em Curitiba. Curitiba: IPPUC, 1996. 158p.

MALUF, R.S.; MENEZES, F. e VALENTE, F.L. Contribuição ao tema de segurança alimentar no Brasil. Cadernos de Debate, Campinas, v. 4, p. 66-88, 1996.

MORLEY, J. E.; GLICK, Z.; RUBENSTEIN, L. Z. Geriatric Nutrition: a comprehensive review. 2. ed. New York: Raven Press, 1995. 397p.

OTERO, U. B. et al. Mortalidade por desnutrição em idosos, região Sudeste do Brasil, 1980-1997. Revista de Saúde Pública, São Paulo, v. 36, n. 2, p. 141-148, 2002.

PENTEADO, P.T.P.S. e OLIVEIRA, K.S. Condições de vida, saúde e nutrição do idoso: Metodologia de entrevista. Curitiba, 50p. 2003

PENTEADO, P.T.P.S. Idoso: condições de vida, saúde e nutrição, no município de Curitiba. Curitiba, 2001, 222f. Tese (Doutorado em Meio Ambiente e Desenvolvimento) - UFPR.

ROSEMBERG, I. H.; MILLER, J. W. Nutritional factors in physical and cognitive functions of elderly people. American Journal of Clinical Nutrition, Bethesda, v. 55, p. 1237S-1243S, 1992.

SICHIERI, R. Epidemiologia da Obesidade. Coleção Saúde e Sociedade, Rio de Janeiro: UERJ, 1998.

STAEHELIN, H. B. Nutrition and ageing. EAMA VI/2. Disponível em: <http://www.healthandage.com

/html/min/eama/publi/0101020035/sld001.htm> Acesso em: 27 out. 2002.

UFPR. UNIVERSIDADE FEDERAL DO PARANÁ. Entrevistas: Estudo multicêntrico sobre estado nutricional e consumo alimentar. Parte 02. Curitiba: UFPR/ Departamento de Nutrição/ INAN. 15p. [1996?].

VELLAS, B. J.; ALBAREDE, J.; GARRY, P. L. Diseases and aging: patterns of morbidity with age; relationship between aging and age-associated diseases. American Journal of Clinical Nutrition, Bethesda, v. 55, p. 1225S-1230S, 1992.

Apoio: Fundação Araucária (PR), Plano Sul-CNPq, UFPR-BANPESQ 098005569, Organização Panamericana de Saúde (OPS). 